\title{
EDITORIAL
}

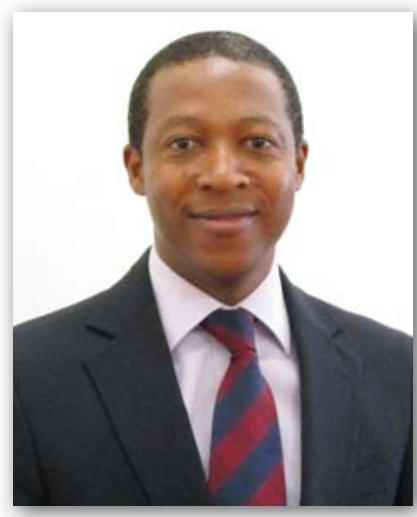

Editor, Ntobeko A.B. Ntusi

Professor of Medicine; Chair and Head, Department of Medicine, University of Cape Town and Groote Schuur Hospital, Observatory, South Africa

\section{Cardiovascular disease in sub-Saharan Africa}

Globally, non-communicable diseases (NCDs) are the leading cause of death, accounting for nearly three-quarters of all deaths, led by cardiovascular diseases (CVD) - with ischaemic heart disease (IHD) and stroke being the leading cause of global cardiovascular (CV) mortality and a major cause of CV morbidity and disability.(I) CVD is an important cause of premature mortality and rising healthcare costs in all regions of the world; and is driven by rapidly evolving cardiometabolic, behavioural, psychological, environmental, and social risk factors and determinants of health.(2) Consistent, comparable, and systematic analysis of trends and patterns in CVD are essential to guide public policy and provide benchmarks for decision-makers.

Worldwide, prevalent cases of total CVD nearly doubled from 27I million in 1990 to 523 million in 2019, and the number of CVD deaths steadily increased from 12.1 million in 1990, reaching 18.6 million in 2019. The global trends for disability-adjusted life years (DALYs) and years of life lost also increased significantly, and years lived with disability doubled from 17.7 million to 34.4 million over that period. The total number of DALYs due to IHD has risen steadily since 1990 , reaching 182 million DALYs, 9.14 million deaths in the year 2019, and 197 million prevalent cases of IHD in 2019. The total number of DALYs due to stroke has risen steadily since 1990, reaching 143 million DALYs, 6.55 million deaths in the year 2019, and 101 million prevalent cases of stroke in 2019.(1)

Sub-Saharan Africa (SSA) is unique epidemiologically. Unlike in every other region in the world, NCDs are the second most common cause of death in SSA, accounting for 2.6 million deaths (about 35\% of all deaths in SSA). ${ }^{(3)}$ In fact, in SSA, the leading cause of mortality is the composite of infections, maternal, neonatal, and nutritional diseases. Current predictions are that by the year 2030, NCDs will account for more than half of all deaths in SSA, and will be the leading cause of mortality, similar to other parts of the world.(4) While age-adjusted CVD mortality rates in SSA are much lower compared to high-income countries, the absolute number of CVD deaths has increased by more than $50 \%$ in the past 3 decades in SSA. ${ }^{(5)}$

\section{HEART FAILURE}

Although there have been no population-based epidemiological studies of heart failure in Africa, there have been numerous hospital-based studies that give important insights into the incidence and prevalence of HF in SSA. ${ }^{(6-12)}$ A recent meta-analysis of heart failure studies in SSA depicted 
that hypertensive heart disease (39\%) was the most common cause of heart failure in SSA, followed by cardiomyopathy (21\%) and rheumatic heart disease - RHD (I4\%), with ischaemic heart disease (IHD) less frequent (7\%). ${ }^{(13)}$ In the THESUS-HF study, conducted in 9 African countries, heart failure was most commonly due to hypertension (45\%), followed by RHD (I 4\%), while IHD accounted for a lower percentage ( 8\%). ${ }^{(8)}$ Similarly, hypertension was the most common cause of heart failure in the Heart of Soweto study. ${ }^{(7)}$ A more contemporaneous study of heart failure in Africa including many patients from private hospitals reported IHD to account for $20 \%$ of heart failure hospitalisations, indicating evidence of epidemiological transition in urban centres. ${ }^{(9)}$

CVD and heart failure in Africa has traditionally been described as distinct to the profile seen in high-income countries, and as having the following characteristics: (i) occurring at a relatively young age and holding great public health relevance because of its high prevalence and impact on young economically active individuals, resulting in significant disability, premature death, and loss of economic productivity; (ii) largely due to non-ischaemic causes, with hypertensive heart disease, rheumatic heart disease (RHD) and cardiomyopathy accounting for majority of cases; (iii) heart failure, rather than IHD, is the dominant form of CVD in SSA; and common causes like $\mathrm{RHD}$, idiopathic cardiomyopathy, peripartum cardiomyopathy, hypertensive heart disease and endomyocardial fibrosis present for the most part in the young; and (iv) infections remain a significant cause of CVD in Africans - pulmonale and pericarditis, largely due to tuberculosis, are important causes of heart failure in the region, while myocarditis, HIV, malaria and syphilis all contribute to the burden of CVD in the region. ${ }^{(6)}$

\section{HYPERTENSION}

In SSA, hypertension is a common medical condition of immense economic importance because of its high prevalence in urban areas, its frequent underdiagnosis, and the severity of its complications. ${ }^{(14)}$ More than a quarter of the global adult population has hypertension and this percentage is expected to increase in the coming years in all regions, particularly in SSA. ${ }^{(15)}$ Prevalence of hypertension in SSA is approximately $30 \%$.(16) There were approximately 80 million patients with hypertension in SSA in 2000, and projections based on current epidemiologic data suggest that this figure will rise to over 150 million by 2025.(17) The highest age-standardised rates of disability-adjusted life years (DALYs) due to hypertension are noted in SSA.(1) An overrepresentation of DALYs due to hypertensive heart disease in African women is noted in Central and Eastern SSA. ${ }^{(1)}$ Inequalities in access to both primary and secondary prevention are a potential cause of these regional and sex differences. Contemporary trends show regional variations with prevalence being associated with the rate of urbanisation and westernisation of lifestyle. ${ }^{(6)}$ The global and regional prevalence of hypertensive heart disease and absolute rates of adverse outcomes are expected to continue to rise due to population growth, aging, increasing obesity, left ventricular hypertrophy and diabetes. ${ }^{(1,6)}$ In black Africans, perhaps due to delayed and/or inadequate therapeutic management and to a likely genetic predisposition, organ-related complications are more common and occur earlier. ${ }^{(6)}$ Stroke, heart failure and renal failure are frequent complications in young African patients, with significant social and economic implications. Intensified global and regional efforts to lower blood pressure and control other risk factors are needed, for example with population-wide reductions in dietary sodium intake. ${ }^{(1)}$ 
Editor,

Ntobeko A.B. Ntusi

\section{RHEUMATIC HEART DISEASE}

Whereas Africa has 10\% of the world's population, as many as half of the 2.4 million children affected by RHD globally live on the continent. ${ }^{(6)}$ RHD accounts for a major proportion of all CVD in children and young adults in African countries, and for 17\% - 43\% of all CVD in SSA, and over 300000 deaths annually. ${ }^{(18)}$ Global age-standardised mortality due to RHD decreased by $48 \%$ from 1990 - 2015, but large differences were observed across regions. In 2015, the highest age-standardised mortality due to and prevalence of RHD was observed in Oceania, South Asia, and Central SSA. In 20I5, there were 33.4 million cases of RHD and I0.5 million DALYs from RHD globally. ${ }^{(18)}$

\section{CARDIOMYOPATHY}

Cardiomyopathy in Africa may be endemic, as in dilated cardiomyopathy (DCM), peripartum cardiomyopathy (PPCM) and endomyocardial fibrosis, or to be non-endemic, as in hypertrophic cardiomyopathy (HCM), arrhythmogenic cardiomyopathy, and HIV-associated cardiomyopathy. Idiopathic DCM contends with hypertension and RHD as the leading causes of heart failure in Africa, accounting for 10\% - 17\% of all cardiac conditions encountered at autopsy and $20 \%$ $30 \%$ of cases of hospitalised heart failure in Africans. ${ }^{(6)}$ In SSA, DCM occurs commonly in the third and fourth decades of life, with men affected twice as much as women. Two-thirds of African patients with DCM, especially those who are more than 55 years of age, have persistently low arterial blood pressure, ventricular arrhythmias and/or atrioventricular valve incompetence and die within 5 years of their first symptom. ${ }^{(19)}$

\section{ISCHAEMIC HEART DISEASE}

Numerous historic reviews and reports have highlighted the rarity of IHD in SSA. In a study conducted in 1960, IHD was reported to be "extremely rare" in Africans, ${ }^{(20)}$ and a separate report declared Africans to be "virtually free" from CAD. (21) Between 1992 and 1994, the Chris Hani Baragwanath Hospital reported a prevalence of $0.2 \%$ of IHD. (22) More recently, investigators of the Heart of Soweto demonstrated evidence of strong epidemiological transition occurring in urban Africans, with consequent "broadened complexity and spectrum of heart disease" in this group, with 10\% of patients studied diagnosed with CAD.(7) Modifications in lifestyle, risk-prone behaviour, diet, cultural attitudes, limited physical activity and other consequences of rapid urbanisation contribute to this epidemiological change.

\section{CONGENITAL HEART DISEASE}

The estimated prevalence of congenital heart disease (CHD) of 8 per 1000 live births is generally accepted as the most reliable globally, though much lower prevalence is observed in Africa, particularly among low-income populations, likely reflecting paucity of readily available estimates and the urgent need for robust data to support burden of disease calculations in Africa. ${ }^{(23)}$ In addition, children with CHD in Africa have a poorer prognosis compared to those from high-income countries. ${ }^{(24)}$ Untreated CHD contributed to the burden of paediatric heart failure in the region.

In this issue of the Journal, we include publications on hypertension, ${ }^{(25)}$ valvular heart disease interventions, ${ }^{(26)} \mathrm{HD}$ diagnosis, ${ }^{(27,28)}$ RHD surgery, ${ }^{(29)}$ HIV associated myocarditis and cardiomyopathy, ${ }^{(30)}$ genomics of $\mathrm{CHD},{ }^{(31)}$ and the role of cardiovascular magnetic resonance in the diagnosis and prognostication of HCM. ${ }^{(32)}$ Obstacles hampering primary and secondary prevention of CVDs in SSA include insufficient healthcare systems and infrastructure, scarcity of cardiac professionals, skewed budget allocation and disproportionate prioritisation away from NCDs, high cost of cardiac treatments and interventions coupled with rarity of health insurance systems. There is much work to be done if we are to overcome these barriers. 
I. Roth GA, Mensah GA, Johnson CO, et al. for Global burden of disease/National Heart Lung and Blood Institute/Journal of the American College of Cardiology global burden of cardiovascular diseases writing group. Global burden of cardiovascular diseases and risk factors, 1990-2019: Update From the GBD 2019 Study. I Am Coll Cardiol 2020;76(25):2982-3021.

2. Vos T, Lim SS, Abbafati C, et al. for the Global burden of diseases 2019 diseases and injuries collaborators. Global burden of 369 diseases and injuries in 204 countries and territories, 1900-2019: A systematic analysis for the Global burden of diseases study 2019. Lancet 2020;396(10258): 1 204-1222.

3. World Health Organisation (WHO). Global health estimates 2016: Deaths by cause, age, sex, by country and by region, 2000-201 6. Geneva: World Health Organisation; 2018. Accessed 23 May 2021. Available at: https://www.who.int/healthinfo/global_burden_disease/estimates/en/indexl.html.

4. Mathers CD, Loncar D. Projections of global mortality and burden of disease from 2002 to 2030. PLoS Medicine 2006; 3(II): e442.

5. Mensah GA, Roth GA, Sampson UK, et al. for the global burden of diseases 2013 mortality and causes of death collaborators. Mortality from cardiovascular diseases in sub-Saharan Africa, 1990-2013: A systematic analysis of data from the global burden of disease study 2013. Cardiovasc J Afr 2015;26(2 Suppl I):S6-SI0.

6. Ntusi NAB, Mayosi BM. Epidemiology of heart failure in sub-Saharan Africa. Expert Rev Cardiovasc Ther 2009; $7(2): 169-180$.

7. Sliwa K, Wilkinson D, Hansen C, et al. Spectrum of heart disease and risk factors in a black urban population in South Africa (the Heart of Soweto Study): A cohort study. Lancet 2008;37। (9616):915-922.

8. Damasceno A, Mayosi BM, Sani M, et al. The causes, treatment, and outcome of acute heart failure in I 006 Africans from 9 countries. Arch Intern Med 2012;72( I8):1 386-1394.

9. Dokainish $\mathrm{H}$, Teo K, Zhu J, et al. Heart failure in Africa, Asia, the Middle East and South America: The INTER-CHF study. Int J Cardiol 2016;204:133-141.

10. Bloomfield GS, Barasa FA, Doll JA, et al. Heart failure in sub-Saharan Africa. Curr Cardiol Rev 20। 3;9(2):157-I73.

11. Sliwa K, Mayosi BM. Recent advances in the epidemiology, pathogenesis and prognosis of acute heart failure and cardiomyopathy in Africa. Heart 2013;99(18):1317-1322.

12. Kraus SM, Ogunbanjo G, Sliwa K, et al. Heart failure in sub-Saharan Africa: A clinical approach. S Afr Med J 2016; 106(I):23-31.

13. Agbor VN, Essouma M, Ntusi NAB, et al. Heart failure in sub-Saharan Africa: A contemporaneous systematic review and meta-analysis. Int J Cardiol 2018;257: 207-215.

14. Bosu WK, Reilly ST, Aheto JMK, et al. Hypertension in older adults in Africa: A systematic review and meta-analysis. PLOS ONE 2019;14(4):e0214934.

15. Sharma JY, Mabhida SE, Myers B, et al. Prevalence of hypertension and its associated risk factors in a rural black population of Mthatha town, South Africa. Int J Environ Res Public Health 2021; 8:1215.

16. Addo J, Smeeth L, Leon DA. Hypertension in sub-Saharan Africa: a systematic review. Hypertens 2007;50:1012-1018.

17. Ezzati M, Lopez AD, Rodgers A, et al. Selected major risk factors and global and regional burden of disease. Lancet 2002;360: 1347- 1360.

18. Watkins DA, Johnson CO, Colquhoun SM, et al. Global, regional, and national burden of rheumatic heart disease, 19902015. N Engl J Med 2017;377:713-722.

19. Chetty S, Mitha AS. Arrhythmias in 88 idiopathic dilated cardiomyopathy: A preliminary study. S Afr Med J 1990; 77:190-193.

20. Shaper AG, Williams AW. Cardiovascular disorders at an African hospital in Uganda. Trans Roy Soc Trop Med Hyg 1960;54:12-32.

21. Vaughan JP. A brief review of cardiovascular disease in Africa. Trans Roy Soc Trop Med Hyg 1977;71:226-23I.

22. Annual Report of the Medical Officer of Health. City of Johannesburg, South Africa (1994).

23. Zühkle L, Mirabel M, Marijon E. Congenital heart disease and rheumatic heart disease in Africa: Recent advances and current priorities. Heart 2013;99(21):|554-1561.

24. Mocombi AO, Lameira E, Yaksh A, et al. Challenges on the management of congenital heart disease in developing countries. Int J Cardiol 201 I; 148:285-288.

25. Ojji D, Ajanya $O$, Abdullahi B, et al. Use of single pill anti-hypertensive combination medications in cardiology clinic of a tertiary health institution in Nigeria. S Afr Heart J 2021; | 8(2):84-87.

26. Du Toit R, Doubell AF, Abelson M, et al. The South African SHARE-TAVI registry: Incidence and risk factors leading to conduction disturbances requiring permanent pacemaker implantation. S Afr Heart J 202 I; | 8(2):88-95.

27. Premsagar P, Aldous $C$, Esterhuizen $T$. The value of exercise stress testing in prediction of angiography amongst South African patients using quantitative scoring systems. S Afr Heart J 2021;18(2):96-106.

28. Premsagar P, Aldous C, Esterhuizen T. A comparison of coronary angiography and myocardial perfusion studies, using quantitative scoring systems. S Afr Heart J 2021;18(2)। 08-116.

29. Tabane M-M T, Kleyenstuber T, Leonard T. Peri-operative outcomes of mitral valve surgery at Charlotte Maxeke Johannesburg Academic Hospital. S Afr Heart J 2021; I 8(2): I 18- 25.

30. Robbertse P-P S, Doubell AF, Nachega JB. The hidden continuum of HIV-associated cardiomyopathy: A focussed review with case reports. S Afr Heart J 2021;18(2): I26-135.

31. Saacks NA, Spracklen TF, Eales JM, et al. The genetics of congenital heart disease: The role of advanced genomic approaches. S Afr Heart J 2021; 18(2): 136-147.

32. Thadathilankal-Jess J. Critical thinking in cardiovascular imaging: Hypertrophic cardiomyopathy and anterior mitral valve cleft. S Afr Heart J 2021; 18(2): 150-I51. 